\title{
Design of Interactive Information Perception System for Internet of Things under 5 G Mobile Communication Technology
}

\author{
Ding Nan \\ Deparment of Electronic information Engineering, Guangzhou College of Technology and Business, \\ Guangzhou, Guangdong, 510850, China \\ email: dingnan143@126.com
}

Keywords: 5G, Mobile Communications Technology, Internet of Things, Information Interaction Technology, Perception Technology, System Design

\begin{abstract}
China's economic and social development can not be separated from the support of information technology. ushered in the rapid upgrading of mobile communication technology, the $5 \mathrm{~g}$ era is coming, the Internet of things in such a technology environment to play a better role in the application field, we must actively explore interactive technology and perception technology, make the Internet of things application field popular and play a better role, to create a more convenient living environment for people. This paper focuses on the design of Internet of things information interactive sensing system under 5G mobile communication technology.
\end{abstract}

\section{Introduction}

The continuous innovation of science and technology has given new development space and ushered in opportunities in various fields. Especially with the $5 \mathrm{G}$ era, mobile communication technology to achieve a new generation, the Internet of things to adapt to the current technology environment to play a better role, from the point of view of application in the technical level of rapid upgrading and continuous improvement, so that it becomes an important part of people's production and life. The Internet of things technology integrates interactive technology and perception technology to realize information interactive transmission, coupled with the application of 5G mobile communication technology, can improve the efficiency of information operation and achieve real-time transmission and interaction, which is more in line with the current rapid life, and the coverage of Internet of things technology can be extended to play its due value.

\section{Basic Understanding of 5G Mobile Communication Technology}

The so-called 5G mobile communication technology is the fifth generation mobile communication technology, which is an extension of the $4 \mathrm{G}$ mobile communication technology. The technology has more coverage and good security, and solves the problems existing in the 4G mobile communication technology, which makes the current social mobile network run more smoothly and meets the needs of users.5 G can be described as an important reform in the history of mobile communication technology, which has high utilization efficiency for spectrum, and the application of high-frequency spectrum resources is more extensive, and the application value is fully reflected. From the performance of $5 \mathrm{G}$ mobile communication technology communication system, multiple application fields can cooperate with each other, or even network each other, and the performance of communication system can be improved. $5 \mathrm{G}$ mobile communication technology embodies the indoor wireless network performance, that is, the coverage performance is more adequate, the service support ability has also been enhanced.5 G mobile communication technology has low energy consumption. When applying 5G mobile communication technology, the operator can adjust the network resources according to the change of traffic, thus realizing the resource optimization and rational utilization, so the operating cost of the technology is relatively low.

5 G mobile communication technology has a good integration, and when it is integrated with 
other wireless communication technologies, a new mobile information network environment will be formed, which is in line with the demand of mobile information network.5 $\mathrm{G}$ mobile communication technology application summary, flexible, and self-adjustment function, in the network environment, self-aware function as an intelligent embodiment, in the application field to show demeanor, Figure 1:5G Mobile Communications Technology Development)

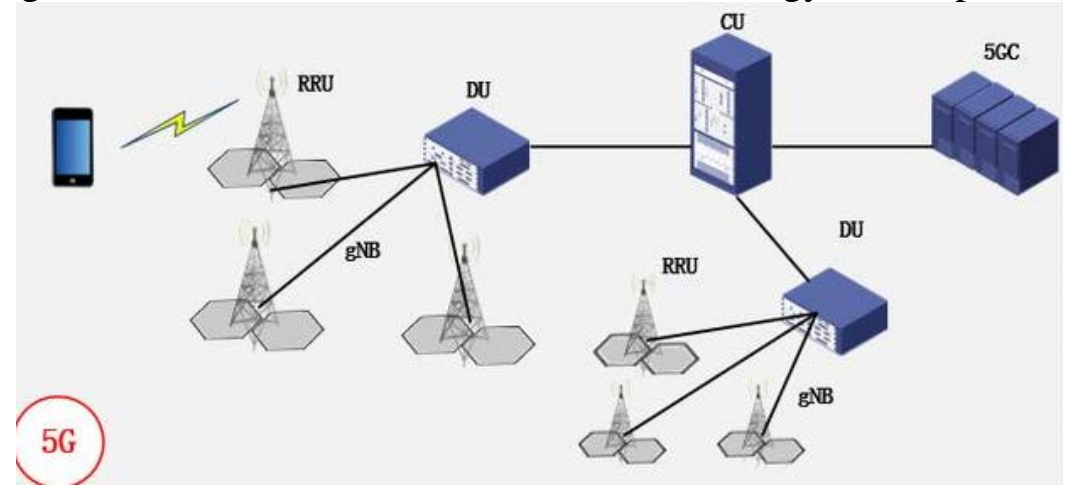

Figure 15 Development of mobile communications technology

\section{Function of the Internet of Things Information Interactive Perception System}

There are differences between Internet of things information interaction perception system and Internet interaction perception technology, the object of Internet of things interaction perception is not restricted, and in Internet of things interaction perception object, one of them is the object, which needs to play the role of intelligent terminal, collect data information, information interaction, realize the interactive perception between people and things, and realize the information transmission between things. In the application of the Internet of things information interaction perception system, the core part is the interaction perception between the user and the network. It is necessary to ensure the interaction technology to play a role and realize the perceived efficiency, so we must do a good job of information transmission control. The relevant contents are as follows.

First, the Internet of things information interaction perception system applications to achieve human-object interaction perception. The Internet is the operation of people and things are indispensable, through the operation of computers, play the role of network technology can achieve data information transmission, in this process, including data identification, detection can be intelligent operation[1]. To ensure the continuous and stable operation of the Internet, but also to do a good job of regular maintenance. The Internet can realize human-computer interaction perception, and the application of relevant technical operations can obtain the information they need, perceive information, store valuable information, and transfer information between clients. The application of interactive sensing technology makes information transmission no longer a single item, but an interactive transmission among users. To ensure the quality of information transmission, we must improve the efficiency of interaction, ensure that the real-time transmission of information, there will be no problem of information loss.

Second, the Internet of things information interactive sensing system applications to achieve network and content interactive perception. The interaction perception between the network and the content is the result of the combination of the Internet of things to the Internet. All kinds of business information are transmitted to customers on the network platform, and the data information is filtered and integrated at the same time, and valuable related data are stored in the specified database. For the storage of data information can be external storage, or can choose the internal storage mode, but these storage methods have defects, the application of distributed database to store information is better. This interactive Cixi transmission is not only fast, efficient, but also low cost.

Third, the Internet of things information interaction perception system applications to achieve user-content interaction perception. The Internet of things realizes the interaction perception between users and content is the embodiment of intelligence, and it provides valuable information 
to meet the needs of users in the course of operation[2]. In the Internet of things, the information interaction between the user and the content plays a bridge role, which makes the user and the content close the distance in the cyberspace, forming a new interaction mode, the user can use the information to retrieve, but also can collect the data information, filter the valuable information, according to the need to replace the information, these work are based on the network intelligent development, not only can the text transmission, the voice interaction, but also the video interaction, the expression interaction between each other to quickly obtain the information they need.(Figure 2: Internet of things information interactive sensing technology application platform)

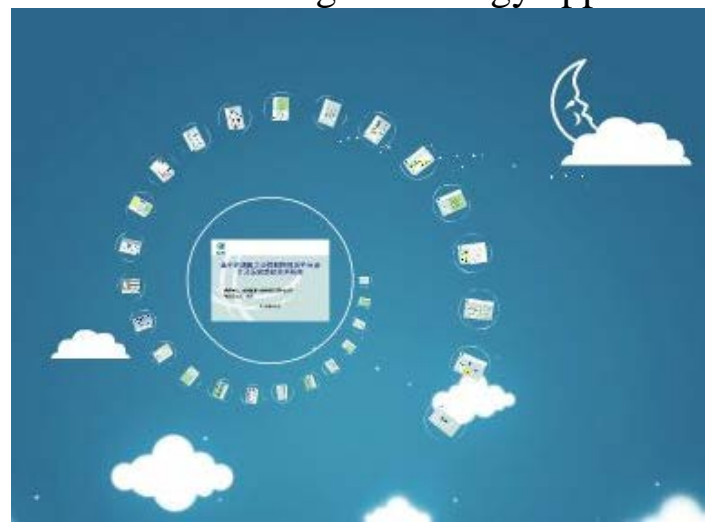

Figure 2 Internet of things information interactive sensing technology application platform

\section{An Effective Scheme for Designing Interactive Information Perception System for Internet of things under 5G Mobile Communication Technology}

\subsection{Building a Management Platform using 5G Mobile Communication Technology}

In the construction of management platform, we should consider the diversity of functions to meet the needs of the current application field, and realize active service perception when providing services to users. In general, when industrial enterprises build management platform, they need to design various types of subsystems to enhance the performance of the platform. In the design of the management platform when the application of $5 \mathrm{G}$ mobile communication technology, to do a good job of network testing, before the implementation of the design work. In the specific design process, we should start from the point of view of energy service demand, consider the actual situation, study the current operation of the platform and the future development trend, adopt advanced technology to establish the platform, ensure the platform operation has good stability, and provide users with high quality service[3]. In the design of the management platform, play the role of 5G communication technology, make the operation intelligent realization, provide a strong support for the management platform, build the intelligent transformation system of resources, especially the speed of user response, so that the value of information collection can be brought into full play, Figure 3: Internet of Things under 5G Mobile Communications)

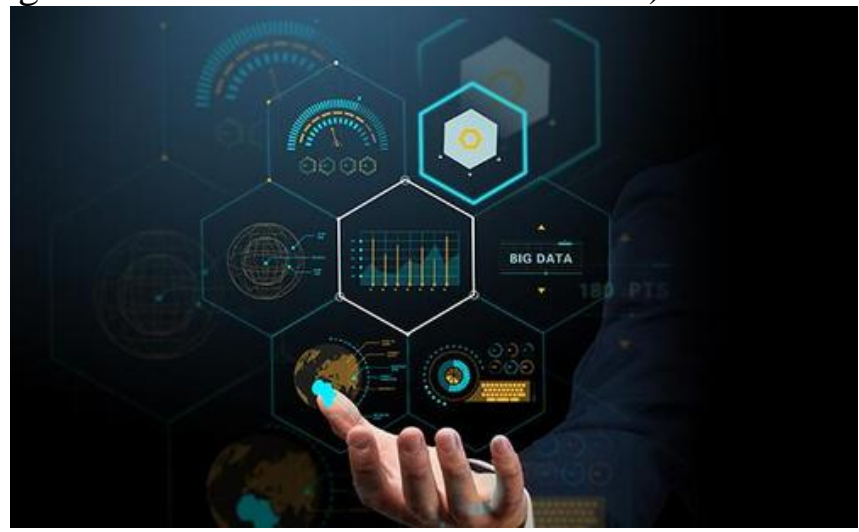

Figure 35 Internet of things under mobile communications technology 


\subsection{Application of 5G Mobile Communication Technology to Construct a Shared Platform}

Using 5G communication technology to construct the service-aware sharing platform, the users are divided into different levels in the operation of the platform, thus providing personalized services for users. The shared platform built in 5G environment draws the distance between the user and the platform, and many services are carried out at the same time to realize the whole scene construction. The application of $5 \mathrm{G}$ mobile communication technology to build a shared platform has more active service perception. In the process of collecting information data, the function of "active service perception" can not only collect information, but also ensure that the information collected has high value, which makes the proposed service scheme more scientific and reasonable, and is feasible in the field of application[4]. With the application of 5G mobile communication technology, the range of data collection can be expanded, in addition to collecting user demand information, including market environment, enterprise operation and other information can be obtained in a timely and accurate manner, details of information, such as system load, equipment operation status, resource allocation, extended data access mode, data transmission security and so on.

\section{Conclusion}

Through the above research can be clear, the current 5G mobile communication era, the Internet of things technology will increase innovation. In order to promote the booster role of Internet of things technology in the development of social economy, it is necessary to actively use information perception technology and interactive technology from the perspective of application to realize the rapid upgrading of technology. Among them, the Internet of things information interaction technology is based on the network technology, can realize the information exchange, the user's data letter also can exchange, brings many conveniences to the application. In the application of information perception technology, it is necessary to collect, filter and analyze the data information and pass it to the specified client, and integrate management for all the information. The current Internet of things technology is still in the development period, because of the existence of this many imperfections, there are still some problems in the design, so it can not be popularized in the field of application. This needs to explore the perception technology and interactive technology extremely, strengthen the design perception of interactive perception system, make the Internet of things technology play a better role in the application field.

\section{Acknowledgements}

F-2019"electronic information engineering", a characteristic specialty construction project of Guangzhou Industrial and Commercial University (item number: TS201902).

\section{References}

[1] Qiu Bo. Discussion on iot technology supported by $5 \mathrm{~g}$ mobile communication. Western Leather, no. 10, pp. 71-71, 2019.

[2] Liu Li, Liu Chunyang, Zhang Chunwang. 5G communication technology to promote the development of Internet of things industry chain. Digital Communications World, no. 12, pp. 47-48, 2018.

[3] Huang Kaizhi, Jinliang, Zhongzhou. 5G physical layer security technology communications for security. ZTE Technologies, no. 04, pp. 25-26, 2019.

[4] Zhang Minggang. On the Key Technologies of 5G Mobile Communication Network. Digital Communications World, no. 03, pp. 98-99, 2018. 